\title{
Research Article \\ Stabilization of Time-Varying System by Controllers with Internal Loop
}

\author{
Yufeng Lu and Chengkai Shi \\ School of Mathematical Sciences, Dalian University of Technology, Dalian 116024, China \\ Correspondence should be addressed to Chengkai Shi, shichengkai2001@163.com
}

Received 18 April 2012; Accepted 10 May 2012

Academic Editor: Zidong Wang

Copyright (c) 2012 Y. Lu and C. Shi. This is an open access article distributed under the Creative Commons Attribution License, which permits unrestricted use, distribution, and reproduction in any medium, provided the original work is properly cited.

We study the concept of stabilization with internal loop for infinite-dimensional discrete timevarying systems in the framework of nest algebra. We originally give a parametrization of all stabilizing controllers with internal loop, and it covers the parametrization of canonical or dual canonical controllers with internal loop obtained before. We show that, in practical application, the controller with internal loop overcomes the awkwardness brought by the extra invertibility condition in the parametrization of the conventional controllers. We also prove that the strong stabilization problem can be completely solved in the closed-loop system with internal loop. Thus the advantage of the controller with internal loop is addressed in the framework of nest algebra.

\section{Introduction}

The closed-loop system whose stability is achieved by the controller with internal loop has attracted the attention of many authors in recent years (see [1-5]). This system was originally introduced by Weiss and Curtain in 1997 in [1]. When they extended the theory of dynamic stabilization to regular linear systems (a subclass of the well-posed linear systems), it was shown in Example 6.5 of [1] that even the standard observer-based controller is not a well-posed linear system as needed, correspondingly, its transfer function is not wellposed. To overcome this difficulty, a new type of controller, the so-called stabilizing controller with internal loop, was introduced. This controller is more general and useful than the standard feedback controller. Until now, only a special class of stabilizing controllers with internal loop called canonical controllers is widely investigated. In [1], a procedure was developed to design the canonical controllers for stabilizable and detectable plants. In [6], the parametrization for all canonical controllers is given which is clean and avoids the extra invertibility condition in the parametrization for the controller in the standard feedback 
system. In [7], the author extended the theory to non-well posed systems, and the robust stabilization problem is considered by using the canonical controller.

In recent years, the study of time-varying systems using modern mathematical methods has come into its own. This was a scientific necessity. After all, many common physical systems are time varying. In [8], A. Feintuch specifically introduced a framework of nest algebra and the control theory for linear time-varying systems was studied in this framework. Meanwhile, many stabilization problems for various nonlinear time-varying systems were widely considered as well (see [9-16]). Based on these cases, we are motivated to consider the new model of closed-loop feedback system with internal loop for time-varying systems.

In this paper, we study the concept of stabilization with internal loop for the linear time-varying system under the framework of nest algebra. We extend our study of controllers with internal loop to more general cases and originally give a parametrization of all stabilizing controllers with internal loop. It is found that the parametrization of the canonical controller obtained in [6] can be viewed as a special case of the parametrization obtained here. As we know, the parametrization of the conventional controller is not clean, and there is always an extra invertibility condition on the parameter. This in turn makes it awkward to use this parametrization to solve the practical problems. While the controller with internal loop overcomes this awkwardness. We take the sensitivity minimization problem as an example to show this advantage of the controller with internal loop. The strong stabilization problem is known as the design of a stable controller which stabilizes the given plant. In the framework of nest algebra, it is still an open problem and only a necessary condition is addressed in [17] for this problem. We prove that any stabilizable plant can be strongly stabilized by the controller with internal loop. This means that the strong stabilization problem can be completely solved in the system with internal loop. We also give a simple example to show how to design the strongly stabilizing controller with internal loop.

This paper is organized as follows. In Section 2, we recall some basic concepts of the linear systems in the framework of nest algebra. In Section 3, we introduce the closed-loop system whose stability is achieved by the controller with internal loop and firstly give a parametrization for all stabilizing controllers with internal loop. In Section 4, we focus on the canonical controller and show the benefit of the controller with internal loop in the practical application. In Section 5, we define the strongly stabilizing controller with internal loop and address an advantage of the controller with internal loop in the framework of nest algebra.

\section{Preliminaries}

Let $\mathscr{\ell}$ be the complex infinite dimensional Hilbert sequence space:

$$
\ell^{2}=\left\{\left(x_{0}, x_{1}, x_{2}, \ldots\right): x_{i} \in \mathbb{C}, \sum_{i=0}^{\infty}\left|x_{i}\right|^{2}<\infty\right\},
$$

where $|\cdot|$ denotes the standard Euclidean norm on $\mathbb{C}$ with inner product $(x, y)=\sum_{i=0}^{\infty} x_{i} \bar{y}_{i}$. $\mathfrak{t}_{e}$ will denote the extended space:

$$
\mathscr{H}_{e}=\left\{\left(x_{0}, x_{1}, x_{2}, \ldots\right): x_{i} \in \mathbb{C}\right\}
$$




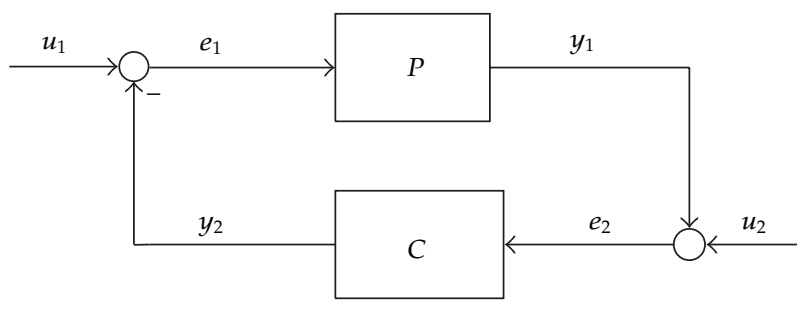

Figure 1: Standard feedback configuration.

For each $n \geq 0$, let $P_{n}$ denote the standard truncation projection defined on $\mathscr{H}$ and $\mathscr{L}_{e}$ by

$$
P_{n}\left(x_{0}, x_{1}, \ldots, x_{n}, x_{n+1}, \ldots\right)=\left(x_{0}, x_{1}, \ldots, x_{n}, 0,0, \ldots\right)
$$

A continuous linear transformation $T$ on $\mathscr{\ell}_{e}$ with the standard seminorm topology ([8, Chapter 5]) is a causal linear system (or a linear system) if for each $n \geq 0, P_{n} T=P_{n} T P_{n}$. Let $\mathcal{L}$ be the set of all linear systems on $\mathcal{d}_{e}$. Then any element of $\mathcal{L}$ is a lower triangular matrix (with respect to the standard basis, see [8, Chapter 5]).

A linear system $T$ is stable if its restriction to $\mathscr{t}$ is a bounded operator ([8, Chapter 5]). We denote the set of stable systems by $\mathcal{S}$, then $\mathcal{S}$ is a weakly closed algebra containing the identity, referred to in the operator algebra literature as a nest algebra ([8, Chapter 5]).

For $P, C \in \mathcal{L}$, we consider the standard feedback configuration with plant $P$ and controller $C$ shown in Figure 1.

$u_{1}, u_{2}$ denote the externally applied inputs; $e_{1}, e_{2}$ denote the inputs to the plant and compensator, respectively, and $y_{1}, y_{2}$ denote the outputs of the plant and compensator, respectively. The closed loop system equations are

$$
\left[\begin{array}{l}
u_{1} \\
u_{2}
\end{array}\right]=\left[\begin{array}{cc}
I & C \\
-P & I
\end{array}\right]\left[\begin{array}{l}
e_{1} \\
e_{2}
\end{array}\right]
$$

The system is well posed if the internal input $e=\left[\begin{array}{l}e_{1} \\ e_{2}\end{array}\right]$ can be expressed as a causal function of the external input $u=\left[\begin{array}{l}u_{1} \\ u_{2}\end{array}\right]$. This is equivalent to requiring that $\left[\begin{array}{cc}I & C \\ -P & I\end{array}\right]$ be invertible. This inverse can be easily computed and is given by the transfer matrix

$$
H(P, C)=\left[\begin{array}{cc}
(I+C P)^{-1} & -C(I+P C)^{-1} \\
P(I+C P)^{-1} & (I+P C)^{-1}
\end{array}\right]
$$

Definition 2.1 (see [8]). The closed loop system $\{P, C\}$ is stable if all the entries of $H(P, C)$ are stable systems on $\mathcal{H}$. The plant $P$ is stabilizable if there exists a causal linear system $C$ such that $\{P, C\}$ is stable.

Recall that the graph of a linear transformation $P$ with domain $\mathscr{\Phi}(P)=\{x \in \mathscr{H}$ : $P x \in \mathscr{L}\}$ is $\mathcal{G}(P)=\left\{\left[\begin{array}{c}x \\ P x\end{array}\right]: x \in \mathscr{D}(P)\right\}$. Then we can give the definitions of strong right representation and strong left representation. 
Definition 2.2 (see [8]). A plant $P$ has a strong right representation $\left[\begin{array}{c}M \\ N\end{array}\right]$ with $M$ and $N$ stable if

(1) $\mathcal{G}(P)=\operatorname{Ran}\left[\begin{array}{c}M \\ N\end{array}\right]$,

(2) there exist $X, Y \in \mathcal{S}$ such that $[Y X]\left[\begin{array}{c}M \\ N\end{array}\right]=I$.

A plant $P$ has a strong left representation $[-\widehat{N} \widehat{M}]$ with $\widehat{M}$ and $\widehat{N}$ stable if

(1) $\mathcal{G}(P)=\operatorname{Ker}[-\widehat{N} \widehat{M}]$,

(2) there exist $\widehat{X}, \widehat{Y} \in \mathcal{S}$ such that $[-\widehat{N} \widehat{M}]\left[\begin{array}{c}-\widehat{X} \\ \hat{Y}\end{array}\right]=I$.

The following result on strong right representation is proved in [8].

Theorem 2.3 (see [8]). Suppose $M, N \in \mathcal{S}$. Then $\left[\begin{array}{c}M \\ N\end{array}\right]$ is a strong right representation of $P \in \mathcal{L}$ if and only if

(1) there exist $X, Y \in \mathcal{S}$ such that $[Y X]\left[\begin{array}{c}M \\ N\end{array}\right]=I$,

(2) $M$ is invertible in $\mathcal{L}$.

We say that a plant $P$ has a right coprime factorization if there exist $M, N, X, Y \in \mathcal{S}$ such that $P=N M^{-1}$ and $Y M+X N=I$. The proof of Theorem 2.1 in [8] implies that $\left[\begin{array}{l}M \\ N\end{array}\right]$ is a strong right representation of $P$ if and only if $N M^{-1}$ is a right coprime factorization of $P$. Similarly, $[-\widehat{N} \widehat{M}]$ is a strong left representation of $P$ if and only if $\widehat{M}^{-1} \widehat{N}$ is a left coprime factorization of $P$.

The following theorem is the classical Youla Parametrization Theorem.

Theorem 2.4 (see [8]). A causal linear system $P \in \mathcal{L}$ is stabilizable if and only if $P$ has a strong right and a strong left representation. If this is the case, the representations can be chosen so that one has the double Bezout identity

$$
\left[\begin{array}{cc}
Y & X \\
-\widehat{N} & \widehat{M}
\end{array}\right]\left[\begin{array}{cc}
M & -\widehat{X} \\
N & \widehat{Y}
\end{array}\right]=\left[\begin{array}{cc}
M & -\widehat{X} \\
N & \widehat{Y}
\end{array}\right]\left[\begin{array}{cc}
Y & X \\
-\widehat{N} & \widehat{M}
\end{array}\right]=\left[\begin{array}{ll}
I & 0 \\
0 & I
\end{array}\right] .
$$

A causal linear system $C$ stabilizes $P$ if and only if it has a strong right representation $\left[\begin{array}{c}\hat{Y}-N Q \\ \hat{X}+M Q\end{array}\right]$ and a strong left representation $[-(X+Q \widehat{M}) Y-Q \widehat{N}]$ for some $Q \in \mathcal{S}$.

\section{Controllers with Internal Loop}

In this section, we investigate the stabilization of the time-varying system by controllers with internal loop in the framework of nest algebra. This system is illustrated in Figure 2.

The intuitively interpretation of Figure 2: $P \in \mathcal{L}$ is the plant and $K_{I}$ is a transfer map from $\left[\begin{array}{l}e_{2} \\ e_{3}\end{array}\right]$ to $\left[\begin{array}{l}y_{2} \\ y_{3}\end{array}\right]$ when all the connections are open. Then the connection from $y_{3}$ to $e_{3}$ is called internal loop. The closed loop system determined by the plant $P$ and the controller $K_{I}$ with internal loop is denoted by $\left\{P, K_{I}\right\}$.

Partitioning $K_{I}$ into

$$
\left[\begin{array}{ll}
C_{11} & C_{12} \\
C_{21} & C_{22}
\end{array}\right]
$$




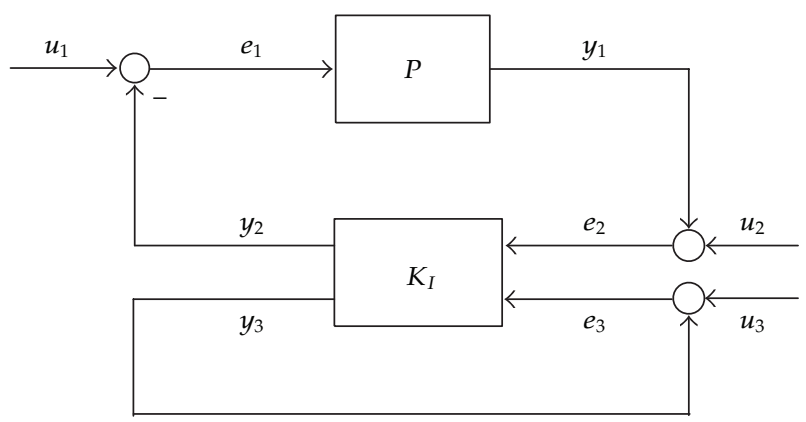

Figure 2: The plant $P$ connected to a controller $K_{I}$ with internal loop.

where $C_{i j} \in \mathcal{L}, i, j=1,2$, the closed loop system equations are

$$
\left[\begin{array}{l}
u_{1} \\
u_{2} \\
u_{3}
\end{array}\right]=\left[\begin{array}{ccc}
I & C_{11} & C_{12} \\
-P & I & 0 \\
0 & -C_{21} & I-C_{22}
\end{array}\right]\left[\begin{array}{l}
e_{1} \\
e_{2} \\
e_{3}
\end{array}\right]
$$

We say that the system is well posed if $\left[\begin{array}{ccc}I & C_{11} & C_{12} \\ -P & I & 0 \\ 0 & -C_{21} & I-C_{22}\end{array}\right]$ is invertible and We denote this inverse by $H\left(P, K_{I}\right)$.

Definition 3.1. The closed loop system $\left\{P, K_{I}\right\}$ determined by the plant $P \in \mathcal{L}$ and the controller with internal loop $K_{I}$ is stable if all the entries of $H\left(P, K_{I}\right)$ are stable. The plant $P$ is stabilizable by a controller with internal loop if there exists a $K_{I}$ such that $H\left(P, K_{I}\right)$ is stable. In this case, $K_{I}$ is called a stabilizing controller with internal loop for $P$.

In the previous papers, the study of stabilizing controller with internal loop is mainly focused on the case that $P$ and $K_{I}$ are both well-posed transfer functions (bounded and analytic on some right half plane). And in all applications, the controller $K_{I}$ is assumed to be stable and satisfy two conditions proposed in [1] (refer to Proposition 4.8 in [1]). While, in the framework of nest algebra, we extend the study to the more general case that $C_{i j} \in \mathcal{L}$, $i, j=1,2$ and $K_{I}$ need not to satisfy the two conditions proposed in [1].

Suppose $I-C_{22}$ is invertible in $\mathcal{L}$, then we have that

$$
H\left(P, K_{I}\right)=\left[\begin{array}{ccc}
(I+C P)^{-1} & -C(I+P C)^{-1} & T_{13} \\
P(I+C P)^{-1} & (I+P C)^{-1} & T_{23} \\
T_{31} & T_{32} & T_{33}
\end{array}\right],
$$

where

$$
\begin{gathered}
C=C_{11}+C_{12}\left(I-C_{22}\right)^{-1} C_{21}, \\
T_{13}=-(I+C P)^{-1} C_{12}\left(I-C_{22}\right)^{-1}, \\
T_{23}=-P(I+C P)^{-1} C_{12}\left(I-C_{22}\right)^{-1},
\end{gathered}
$$




$$
\begin{aligned}
& T_{31}=\left(I-C_{22}\right)^{-1} C_{21} P(I+C P)^{-1}, \\
& T_{32}=\left(I-C_{22}\right)^{-1} C_{21}(I+P C)^{-1}, \\
& T_{33}=\left(I-\left(I-C_{22}\right)^{-1} C_{21} P(I+C P)^{-1} C_{12}\right)\left(I-C_{22}\right)^{-1} .
\end{aligned}
$$

Remark 3.2. Notice that the upper left $2 \times 2$ corner of the above transfer matrix $H\left(P, K_{I}\right)$ is just the transfer matrix $H(P, C)$ of the standard feedback system with the plant $P$ and the controller $C=C_{11}+C_{12}\left(I-C_{22}\right)^{-1} C_{21}$. This implies that the closed-loop system stabilized by controllers with internal loop is more general than the standard feedback system and its transfer matrix provides more information.

Now we can give a parametrization of all stabilizing controllers with internal loop with $I-C_{22}$ invertible in $\mathcal{L}$.

Theorem 3.3. Suppose $P \in \mathcal{L}$ and there exist $M, N, X, Y, \widehat{M}, \widehat{N}, \widehat{X}, \widehat{Y} \in \mathcal{S}$ such that $\left[\begin{array}{c}M \\ N\end{array}\right]$ and $[-\widehat{N} \widehat{M}]$ are, respectively, strong right and left representation for $P$ that satisfy the double Bezout identity

$$
\left[\begin{array}{cc}
Y & X \\
-\widehat{N} & \widehat{M}
\end{array}\right]\left[\begin{array}{cc}
M & -\widehat{X} \\
N & \hat{Y}
\end{array}\right]=\left[\begin{array}{cc}
M & -\widehat{X} \\
N & \hat{Y}
\end{array}\right]\left[\begin{array}{cc}
Y & X \\
-\widehat{N} & \widehat{M}
\end{array}\right]=\left[\begin{array}{ll}
I & 0 \\
0 & I
\end{array}\right] .
$$

Then all stabilizing controllers with internal loop $K_{I}=\left[\begin{array}{ll}C_{11} & C_{12} \\ C_{21} & C_{22}\end{array}\right]$ are parameterized by

$$
\begin{aligned}
C_{11}= & (\widehat{X}+M Q)(\widehat{Y}-N Q)^{-1} \\
& -(Y-Q \widehat{N})^{-1} R_{1}\left(R_{3}+R_{2}(\widehat{Y}-N Q)^{-1} N R_{1}\right)^{-1} R_{2}(\widehat{Y}-N Q)^{-1}, \\
C_{12}= & (Y-Q \widehat{N})^{-1} R_{1}\left(R_{3}+R_{2}(\widehat{Y}-N Q)^{-1} N R_{1}\right)^{-1}, \\
C_{21}= & \left(R_{3}+R_{2}(\widehat{Y}-N Q)^{-1} N R_{1}\right)^{-1} R_{2}(\widehat{Y}-N Q)^{-1}, \\
C_{22}= & I-\left(R_{3}+R_{2}(\widehat{Y}-N Q)^{-1} N R_{1}\right)^{-1},
\end{aligned}
$$

for some $Q, R_{1}, R_{2}, R_{3} \in \mathcal{S}$.

In order to prove this theorem clearly, we need the following result which is an improvement of Theorem 2.4. It is interesting that while the two representations for the controller in Theorem 2.4 are independent, the same $Q$ will in fact work for both.

Theorem 3.4. Suppose P satisfies the assumption in Theorem 2.4. Then the stabilizing controller $C$ for $P$ has the form $C=(Y-Q \widehat{N})^{-1}(X+Q \widehat{M})=(\widehat{X}+M Q)(\widehat{Y}-N Q)^{-1}$ for some $Q \in \mathcal{S}$. 
Proof. Suppose $C$ stabilizes $P$. By Theorem 2.4, we have that $C$ has a right coprime factorization $C=(\widehat{X}+M Q)(\widehat{Y}-N Q)^{-1}$ for some $Q \in \mathcal{S}$. It is easy to check that

$$
\begin{aligned}
{\left[\begin{array}{cc}
-(X+Q \widehat{M}) & Y-Q \widehat{N} \\
\widehat{M} & \widehat{N}
\end{array}\right]\left[\begin{array}{cc}
-N & \widehat{Y}-N Q \\
M & \widehat{X}+M Q
\end{array}\right] } & =\left[\begin{array}{cc}
-N & \widehat{Y}-N Q \\
M & \widehat{X}+M Q
\end{array}\right]\left[\begin{array}{cc}
-(X+Q \widehat{M}) & Y-Q \widehat{N} \\
\widehat{M} & \widehat{N}
\end{array}\right] \\
& =\left[\begin{array}{ll}
I & 0 \\
0 & I
\end{array}\right]
\end{aligned}
$$

Thus,

$$
[-(X+Q \widehat{M}) Y-Q \widehat{N}]\left[\begin{array}{c}
\widehat{Y}-N Q \\
\widehat{X}+M Q
\end{array}\right]=0
$$

This implies that

$$
\mathcal{G}(C)=\operatorname{Ran}\left[\begin{array}{c}
\widehat{Y}-N Q \\
\widehat{X}+M Q
\end{array}\right] \subseteq \operatorname{Ker}[-(X+Q \widehat{M}) \quad Y-Q \widehat{N}]
$$

On the other hand, for any $\left[\begin{array}{l}x \\ y\end{array}\right] \in \operatorname{Ker}[-(X+Q \widehat{M}) \quad Y-Q \widehat{N}]$, we have

$$
\begin{aligned}
& {\left[\begin{array}{l}
x \\
y
\end{array}\right]=\left[\begin{array}{cc}
-N & \widehat{Y}-N Q \\
M & \widehat{X}+M Q
\end{array}\right]\left[\begin{array}{cc}
-(X+Q \widehat{M}) & Y-Q \widehat{N} \\
\widehat{M} & \widehat{N}
\end{array}\right]\left[\begin{array}{l}
x \\
y
\end{array}\right]} \\
& =\left(\left[\begin{array}{c}
-N \\
M
\end{array}\right][-(X+Q \widehat{M}) Y-Q \widehat{N}]+\left[\begin{array}{c}
\widehat{Y}-N Q \\
\widehat{X}+M Q
\end{array}\right]\left[\begin{array}{ll}
\widehat{M} & \widehat{N}
\end{array}\right]\right)\left[\begin{array}{l}
x \\
y
\end{array}\right] \\
& =\left[\begin{array}{l}
\widehat{Y}-N Q \\
\widehat{X}+M Q
\end{array}\right]\left[\begin{array}{ll}
\widehat{M} & \widehat{N}
\end{array}\right]\left[\begin{array}{l}
x \\
y
\end{array}\right] \in \mathcal{G}(C),
\end{aligned}
$$

that is,

$$
\operatorname{Ker}[-(X+Q \widehat{M}) Y-Q \widehat{N}] \subseteq \mathcal{G}(C)
$$

Thus,

$$
\mathcal{G}(C)=\operatorname{Ker}[-(X+Q \widehat{M}) Y-Q \widehat{N}]
$$

Since

$$
[-(X+Q \widehat{M}) Y-Q \widehat{N}]\left[\begin{array}{c}
-N \\
M
\end{array}\right]=I,
$$


we obtain that $[-(X+Q \widehat{M}) Y-Q \widehat{N}]$ is a strong left representation of $C$ and $C=(Y-$ $Q \widehat{N})^{-1}(X+Q \widehat{M})$. This completes the proof.

Now we can give the proof of Theorem 3.3.

Proof of Theorem 3.3. Suppose $\left\{P, K_{I}\right\}$ is stable, then every entry of the matrix

$$
\left[\begin{array}{cc}
(I+C P)^{-1} & -C(I+P C)^{-1} \\
P(I+C P)^{-1} & (I+P C)^{-1}
\end{array}\right]
$$

is in $\mathcal{S}$ and $T_{13}, T_{23}, T_{31}, T_{32}, T_{33} \in \mathcal{S}$. Note that (3.14) is just the transfer matrix $H(P, C)$ for the standard feedback system. By Theorem 3.4, we see that $C$ has the following representation:

$$
C=(Y-Q \widehat{N})^{-1}(X+Q \widehat{M})=(\widehat{X}+M Q)(\widehat{Y}-N Q)^{-1},
$$

for some $Q \in \mathcal{S}$. In this case,

$$
\begin{aligned}
T_{13} & =-(I+C P)^{-1} C_{12}\left(I-C_{22}\right)^{-1} \\
& =-M(Y-Q \widehat{N}) C_{12}\left(I-C_{22}\right)^{-1} \in \mathcal{S}, \\
T_{23} & =-P(I+C P)^{-1} C_{12}\left(I-C_{22}\right)^{-1} \\
& =-N(Y-Q \widehat{N}) C_{12}\left(I-C_{22}\right)^{-1} \in \mathcal{S},
\end{aligned}
$$

if and only if

$$
(Y-Q \widehat{N}) C_{12}\left(I-C_{22}\right)^{-1} \in \mathcal{S}
$$

It follows that

$$
C_{12}\left(I-C_{22}\right)^{-1}=(Y-Q \widehat{N})^{-1} R_{1}
$$

for some $R_{1} \in \mathcal{S}$.

In the same way, we obtain that

$$
\left(I-C_{22}\right)^{-1} C_{21}=R_{2}(\widehat{Y}-N Q)^{-1},
$$

for some $R_{2} \in \mathcal{S}$. So we get

$$
\begin{aligned}
& C_{12}=(Y-Q \widehat{N})^{-1} R_{1}\left(I-C_{22}\right), \\
& C_{21}=\left(I-C_{22}\right) R_{2}(\widehat{Y}-N Q)^{-1} .
\end{aligned}
$$


Since

$$
\begin{aligned}
T_{33} & =\left(I-\left(I-C_{22}\right)^{-1} C_{21} P(I+C P)^{-1} C_{12}\right)\left(I-C_{22}\right)^{-1} \\
& =\left(I-C_{22}\right)^{-1}-R_{2}(\widehat{Y}-N Q)^{-1} N(Y-Q \widehat{N})(Y-Q \widehat{N})^{-1} R_{1} \\
& =\left(I-C_{22}\right)^{-1}-R_{2}(\widehat{Y}-N Q)^{-1} N R_{1} \in \mathcal{S}
\end{aligned}
$$

we have

$$
\left(I-C_{22}\right)^{-1}=R_{3}+R_{2}(\widehat{Y}-N Q)^{-1} N R_{1}
$$

for some $R_{3} \in \mathcal{S}$. Thus,

$$
C_{22}=I-\left(R_{3}+R_{2}(\widehat{Y}-N Q)^{-1} N R_{1}\right)^{-1}
$$

Then we can obtain the following representations for $C_{12}$ and $C_{21}$ :

$$
\begin{aligned}
& C_{12}=(Y-Q \widehat{N})^{-1} R_{1}\left(R_{3}+R_{2}(\widehat{Y}-N Q)^{-1} N R_{1}\right)^{-1}, \\
& C_{21}=\left(R_{3}+R_{2}(\widehat{Y}-N Q)^{-1} N R_{1}\right)^{-1} R_{2}(\widehat{Y}-N Q)^{-1} .
\end{aligned}
$$

Substituting the representations of $C, C_{12}, C_{21}$, and $C_{22}$ into $C_{11}=C-C_{12}\left(I-C_{22}\right)^{-1} C_{21}$, we obtain

$$
\begin{aligned}
C_{11}= & (\widehat{X}+M Q)(\widehat{Y}-N Q)^{-1}-(Y-Q \widehat{N})^{-1} \\
& \times R_{1}\left(R_{3}+R_{2}(\widehat{Y}-N Q)^{-1} N R_{1}\right)^{-1} R_{2}(\widehat{Y}-N Q)^{-1} .
\end{aligned}
$$

This completes the proof.

It was said in [1] that the controller with internal loop was particularly well suited for tracking, and a physical interpretation was given for the system with internal loop. In [6], the author described a seemingly impossible problem, the "intriguing control problem", which can be easily solved by the system with internal loop. In the next two sections, we will show the other great advantages of the controller with internal loop. 


\section{Canonical Controllers and Dual Canonical Controllers}

In this section, we focus on two special classes of controllers with internal loop called canonical controllers and dual canonical controllers, respectively. Here below it is given their definitions in the framework of nest algebra.

Definition 4.1. A controller with internal loop is called the canonical controller for the plant $P$ if it is of the form $K_{I}=\left[\begin{array}{cc}0 & I \\ C_{21} & C_{22}\end{array}\right]$ with $C_{21}, C_{22} \in \mathcal{S}$. Analogously, a controller with internal loop is called a dual canonical controller for the plant $P$ if it is of the form $\left[\begin{array}{lll}0 & C_{12} \\ I & C_{22}\end{array}\right]$ with $C_{12}$, $C_{22} \in \mathcal{S}$.

For canonical controllers, we have the following results.

Theorem 4.2. The canonical controller $K_{I}=\left[\begin{array}{cc}0 & I \\ C_{21} & C_{22}\end{array}\right]$ stabilizes $P \in \mathcal{L}$ with internal loop if and only if $\Delta=I-C_{22}+C_{21} P$ is invertible in $\mathcal{L}$ and $\Delta^{-1}, P \Delta^{-1} \in \mathcal{S}$.

If $P$ has a strong right representation $\left[\begin{array}{c}M \\ N\end{array}\right]$, then the canonical controller $K_{I}$ stabilizes $P$ if and only if $D=M-C_{22} M+C_{21} N$ is invertible in $\mathcal{S}$.

Proof. According to the system equations in (3.2), we have that, for the canonical controllers $K_{I}=\left[\begin{array}{cc}0 & I \\ C_{21} & C_{22}\end{array}\right]$, the transfer matrix $H\left(P, K_{I}\right)$ can be given by

$$
H\left(P, K_{I}\right)=\left[\begin{array}{ccc}
I-\Delta^{-1} C_{21} P & -\Delta^{-1} C_{21} & -\Delta^{-1} \\
P\left(I-\Delta^{-1} C_{21} P\right) & I-P \Delta^{-1} C_{21} & -P \Delta^{-1} \\
\Delta^{-1} C_{21} P & \Delta^{-1} C_{21} & \Delta^{-1}
\end{array}\right] .
$$

Thus, $H\left(P, K_{I}\right) \in M_{3}(\mathcal{S})$ if and only if $\Delta^{-1}, P \Delta^{-1}, \Delta^{-1} C_{21} P$, and $P\left(I-\Delta^{-1} C_{21} P\right)$ are all in $S$. Since $\Delta^{-1} C_{21} P=\Delta^{-1}\left(\Delta+C_{21} P\right)-I=\Delta^{-1}\left(I-C_{22}\right)-I$ and $P\left(I-\Delta^{-1} C_{21} P\right)=P \Delta^{-1}\left(I-C_{22}\right)$. We have that all the entries of $H\left(P, K_{I}\right)$ are in $\mathcal{S}$ if and only if $\Delta^{-1}$ and $P \Delta^{-1}$ are in $\mathcal{S}$. Thus the first statement is proved.

Let us prove the second assertion in the theorem. If $P=N M^{-1}$ and $D^{-1} \in \mathcal{S}$, we have that $\Delta^{-1}=M\left(M-C_{22} M+C_{21} N\right)^{-1}=M D^{-1} \in \mathcal{S}$ and $P \Delta^{-1}=N D^{-1} \in \mathcal{S}$. By using the first result, we have that $K_{I}=\left[\begin{array}{cc}0 & I \\ C_{21} & C_{22}\end{array}\right]$ stabilizes $P$. Conversely, if $K_{I}=\left[\begin{array}{cc}0 & I \\ C_{21} & C_{22}\end{array}\right]$ stabilizes $P$. By the first result, we have that $\Delta^{-1}, P \Delta^{-1}$ are both in $\mathcal{S}$. Suppose that $M, N, X$, and $Y$ are as in Definition 2.2, then

$$
Y \Delta^{-1}+X P \Delta^{-1}=(Y M+X N)\left(M-C_{22} M+C_{21} N\right)=D^{-1} .
$$

Since $X$ and $Y$ are in $\mathcal{S}$, we see that $D^{-1} \in \mathcal{S}$. This completes the proof.

There is a similar result for the dual canonical controller.

Theorem 4.3. The dual canonical controller $K_{I}=\left[\begin{array}{ll}0 & C_{12} \\ I & C_{22}\end{array}\right]$ stabilizes $P \in \mathcal{L}$ with internal loop if and only if $\widehat{\Delta}=I-C_{22}+P C_{12}$ is invertible in $\mathcal{L}$ and $\widehat{\Delta}^{-1}, \widehat{\Delta}^{-1} P \in \mathcal{S}$.

If $P$ has a strong left representation $[-\widehat{N} \widehat{M}]$, then the dual canonical controller $K_{I}$ stabilizes $P$ if and only if $\widehat{D}=\widehat{M}-\widehat{M} C_{22}+\widehat{N} C_{12}$ is invertible in $\mathcal{S}$.

In [6], the parametrization of all canonical controllers and dual canonical controllers is given and it can be easily extended to our framework. 
Theorem 4.4. Suppose $P \in \mathcal{L}$ satisfies the assumption of Theorem 3.3. Then all canonical controllers that stabilize $P$ are parameterized by

$$
\left[\begin{array}{cc}
0 & I \\
E(X+Q \widehat{M}) & I-E(Y-Q \widehat{N})
\end{array}\right]
$$

where $Q \in \mathcal{S}$ and $E$ is invertible in $\mathcal{S}$.

Analogously, all dual canonical controllers that stabilize $P$ are parameterized by

$$
\left[\begin{array}{cc}
0 & (\widehat{X}+M Q) R \\
I & I-(\widehat{Y}-N Q) R
\end{array}\right]
$$

where $Q \in \mathcal{S}$ and $R$ is invertible in $\mathcal{S}$.

Remark 4.5. Indeed, if we choose the parameters in Theorem 3.3 such that $R_{1}=E^{-1}, R_{2}=$ $\widehat{X}+M Q$, and $R_{3}=M E^{-1}$, we can obtain the same result of the above theorem. This implies that the result derived in [6] can be regarded as a special case of Theorem 3.3.

The following theorem gives a strong relation between the stabilization with canonical controller and the usual concept of stabilization.

Theorem 4.6. Suppose $I-C_{22}$ is invertible in $\mathcal{L}$, then $P$ can be stabilized by a canonical controller with internal loop if and only if $P$ is stabilizable in the framework of standard feedback system.

Proof. Suppose $P$ is stabilized by a canonical controller $K_{I}=\left[\begin{array}{ll}0 & C_{12} \\ I & C_{22}\end{array}\right]$ with $I-C_{22}$ invertible in $\mathcal{L}$. Then all entries of $H\left(P, K_{I}\right)$ in (4.1) are in $\mathcal{S}$. By computation, we can easily obtain that the upper left $2 \times 2$ corner of the transfer matrix $H\left(P, K_{I}\right)$ is just the transfer matrix $H(P, C)$ in the standard feedback system with the plant $P$ and the controller $C=\left(I-C_{22}\right)^{-1} C_{21}$. It follows that $P$ is stabilizable in the standard feedback system.

On the other hand, suppose $P$ is stabilizable in the standard feedback system. Then, from Theorem 2.4, all the stabilizing controllers can be given by $C=(Y-Q \widehat{N})^{-1}(X+Q \widehat{M})$ with some $Q \in \mathcal{S}$. Let $C_{21}=X+Q \widehat{M}, C_{22}=Y-Q \widehat{N}$, then we obtain a canonical controller $K_{I}=\left[\begin{array}{cc}0 & I \\ X+Q \widehat{M} & Y-Q \widehat{N}\end{array}\right]$ and it is easy to verify that $K_{I}$ stabilizes $P$.

Naturally, there exists a dual result for the dual canonical controller. application.

Now we can explain the advantage of the controller with internal loop in the practical

Recall the parametrization of the conventional controllers in Theorem 2.4, it is not clean and an extra invertibility condition is imposed on the Youla parameter. This in turn makes it awkward to use this parametrization to solve the practical problems. For example, in [8, Section 7], the sensitivity minimization problem for the system described in Figure 3 is studied. The weighted sensitivity operator for this system is defined by $S_{W}=(I+P C)^{-1} W$ and the weighted sensitivity minimization problem is to find

$$
\inf \left\{\left\|S_{W}\right\|: C \text { stabilizes } P\right\} \text {. }
$$




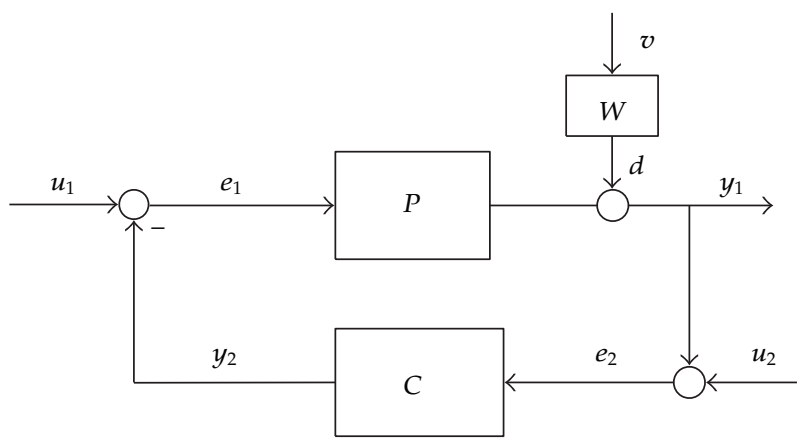

Figure 3: Standard feedback system with outside disturbance $d$ and $W$ invertible.

Suppose $P \in \mathcal{L}$ satisfy the condition in Theorem 2.4 , then all stabilizing controllers can be given by $C=(\widehat{X}+M Q)(\widehat{Y}-N Q)^{-1}$ with $Q \in \mathcal{S}$ and $\widehat{Y}-N Q$ is invertible in $\mathcal{L}$. By simple computation, we can obtain that the weighted sensitivity minimization problem is to find

$$
\inf \{\|\widehat{Y} \widehat{M} W-N Q \widehat{M} W\|: Q \in \mathcal{S}, \widehat{Y}-N Q \text { is invertible in } \mathcal{\perp}\}
$$

Obviously, the extra condition that $\widehat{Y}-N Q$ is invertible in $\mathcal{L}$ makes the practical control engineers difficult to continue their computations. So they have to choose to ignore the fact that the Youla parameter can not be taken for all the elements in $\mathcal{S}$.

Fortunately, the controller with internal loop overcomes this awkwardness. Let us consider the sensitivity minimization problem for the system with internal loop as described in Figure 4. We consider this problem for the dual canonical controller $K_{I}=\left[\begin{array}{ll}0 & C_{12} \\ I & C_{22}\end{array}\right]$ with $C_{12}$, $C_{22} \in \mathcal{S}$. When $u_{1}=u_{2}=u_{3}=0$, it is easy to check that the weighted sensitivity operator for this system is

$$
S_{W}=\left[I-P C_{12}\left(I-C_{22}+P C_{12}\right)^{-1}\right] W
$$

and the weighted sensitivity minimization problem is to find

$$
\inf \left\{\left\|S_{W}\right\|: K_{I}=\left[\begin{array}{ll}
0 & C_{12} \\
I & C_{22}
\end{array}\right] \text { stabilizes } P\right\}
$$

By using the parametrization of the dual canonical controller given in Theorem 4.4, the weighted sensitivity minimization problem is to find

$$
\inf \{\|\widehat{Y} \widehat{M} W-N Q \widehat{M} W\|: Q \in \mathcal{S}\}
$$

Obviously, it avoids the extra invertibility condition for the parameter $Q$ as it appears in the standard feedback system. This overcomes the difficulty arisen in the standard feedback system. 


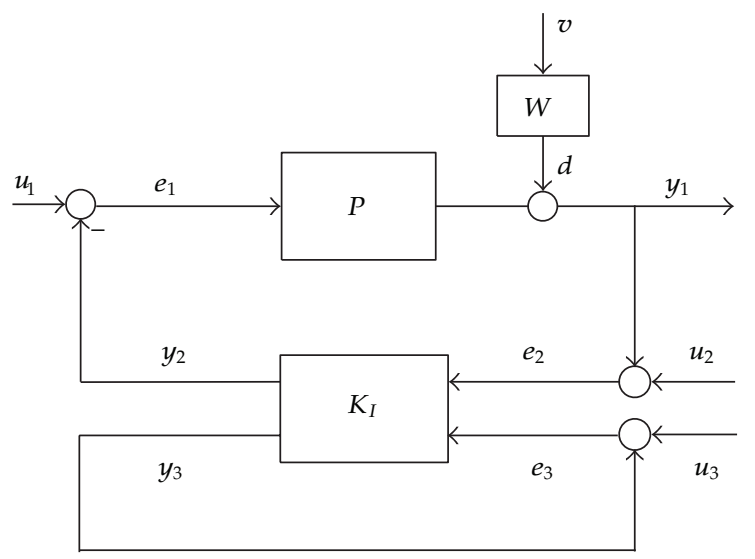

Figure 4: System with internal loop with outside disturbance $d$ and $W$ invertible.

\section{Strong Stabilization with Internal Loop}

Practicing control engineers is reluctant to use unstable compensators for the purpose of stabilization. This motivated considering the strong stabilization problem whether among the stabilizing controllers for a given stabilizable plant $P$, there exist stable ones. If there exists such a controller, $P$ is said to be strongly stabilizable and the stable controller is called the strongly stabilizing controller. In this section, we consider the strong stabilization problem for the system with internal loop and address another advantage of the controller with internal loop.

Definition 5.1. $P \in \mathcal{L}$ is said to be strongly stabilizable with internal loop if it can be stabilized by the controller $K_{I}=\left[\begin{array}{ll}C_{11} & C_{12} \\ C_{21} & C_{22}\end{array}\right]$ with $C_{i j} \in \mathcal{S}, i, j=1,2$. This controller $K_{I}$ is called the strongly stabilizing controller with internal loop.

Obviously, the canonical controller and dual canonical controller are both the strongly stabilizing controller with internal loop. From the parametrization of controllers with internal loop given in Theorem 3.3, we see that the strongly stabilizing controller with internal loop can be characterize by choosing the parameters $Q, R_{1}, R_{2}$, and $R_{3}$ in Theorem 3.3 such that $\widehat{Y}-N Q$ and $R_{3}+R_{2}(\widehat{Y}-N Q)^{-1} N R_{1}$ are invertible in $\mathcal{S}$. The following theorem shows the existence of the strongly stabilizing controller with internal loop.

Theorem 5.2. Suppose $P \in \mathcal{L}$ is stabilizable, then $P$ can be strongly stabilized by the controller with internal loop.

Proof. Suppose $P$ is stabilizable. From Theorem 2.4, the controller stabilizes $P$ has the parametrization that $C=(Y-Q \widehat{N})^{-1}(X+Q \widehat{M})$ for some stable $Q$. Set $C_{11}=0, C_{12}=I$, $C_{21}=(X+Q \widehat{M})$ and $C_{22}=I-(Y-Q \widehat{N})$, then $K_{I}=\left[\begin{array}{cc}0 & I \\ X+Q \widehat{M} I-(Y-Q \widehat{N})\end{array}\right]$ is a stable controller with internal loop and it strongly stabilizes the plant $P$.

Remark 5.3. In [17], it is proved that a given plant $P$ with left coprime factorization $P=\widehat{M}^{-1} \widehat{N}$ can be strongly stabilized in the standard feedback system if $\widehat{N}$ is compact. While in the case where $\widehat{N}$ is not compact, it is still an open problem whether or not there exists a stable 
controller that stabilizes the plant. Theorem 5.2 shows that any stabilizable plant can be stabilized by a stable controller with internal loop. It implies that the strong stabilization problem can be completely solved by the controller with internal loop. And this addresses an advantage of the controller with internal loop in the framework of nest algebra.

From the proof of Theorem 5.2, we see that it also provides a method to design the strongly stabilizing controller with internal loop. We end our paper with a simple example to show this design method.

Example 5.4. Suppose $P=I$, it is obviously stabilizable. Take

$$
\begin{aligned}
& N=\widehat{N}=M=\widehat{M}=I \text {, } \\
& Y=\widehat{Y}=\left[\begin{array}{ccccc}
1 & & & & \\
0 & \frac{1}{2} & & & \\
& 0 & 1 & & \\
0 & 0 & \frac{3}{3} & & \\
0 & 0 & 0 & \frac{1}{4} & \\
\vdots & \vdots & \vdots & \vdots & \ddots
\end{array}\right], \quad X=\widehat{X}=\left[\begin{array}{ccccc}
0 & & & & \\
0 & \frac{1}{2} & & & \\
& 0 & 2 & & \\
0 & 0 & \frac{3}{3} & & \\
0 & 0 & 0 & \frac{3}{4} & \\
\vdots & \vdots & \vdots & \vdots & \ddots
\end{array}\right] \text {. }
\end{aligned}
$$

From Theorem 2.4, we obtain the parametrization of the stabilizing controller $C=(Y-$ $Q \widehat{N})^{-1}(X+Q \widehat{M})$ for some stable $Q$. Take $Q=0$, then

$$
C=Y^{-1} X \text { stabilizes } P \text { and } C=\left[\begin{array}{llllll}
0 & & & & \\
0 & 1 & & & \\
0 & 0 & 2 & & \\
0 & 0 & 0 & 3 & \\
\vdots & \vdots & \vdots & \vdots & \ddots
\end{array}\right]
$$

is unstable. In order to satisfy the practicing control engineers' requirement, we set $C_{11}=0$, $C_{12}=I, C_{21}=X$ and $C_{22}=I-Y$. Then we obtain a stable controller $K_{I}=\left[\begin{array}{cc}0 & I \\ X & I-Y\end{array}\right]$ with internal loop which stabilizes the plant $P$.

\section{Conclusion}

In this paper, the closed-loop system whose stability is achieved by the controller with internal loop is studied in the framework of nest algebra. The controllers with internal loop considered here are more general than those in the previous paper and they are not necessarily stable and need not to satisfy the two conditions proposed in [1]. We give a parametrization for all stabilizing controllers with internal loop which has never been studied before. Then we show that this parametrization covers the parametrization for canonical or dual canonical controllers obtained in [6]. By taking the sensitivity minimization problem as an example, we show that, in the practical application, the controller with internal loop solves the difficulty brought by the invertibility condition in the parametrization of the conventional controller. In the framework of nest algebra, the strong stabilization problem is still an open problem, and no sufficient and necessary condition was found to characterize the plant 
which can be strongly stabilized. While, with the help of the concept of stabilization with internal loop, we show that any stabilizable plant can be strongly stabilized by the controller with internal loop. This addresses an advantage of the controller with internal loop in the framework of nest algebra. By using the parametrization of the controller with internal loop, we are considering other questions in the control theory for the this model of closed-loop systems with internal loop.

\section{Acknowledgments}

The authors would like to thank Doctor Liu Liu for pointing out Lemma 3.1 and Doctor Gong Ting for her invaluable suggestions. This research is supported by NSFC, item number: 10971020.

\section{References}

[1] G. Weiss and R. F. Curtain, "Dynamic stabilization of regular linear systems," Institute of Electrical and Electronics Engineers, vol. 42, no. 1, pp. 4-21, 1997.

[2] R. Curtain, G. Weiss, and M. Weiss, "Coprime factorization for regular linear systems," Automatica, vol. 32, no. 11, pp. 1519-1531, 1996.

[3] S. Townley, G. Weiss, and Y. Yamamoto, "Discretizing continuous time controllers for infinitedimensional linear systems," in Proceedings of the MTNS Symposium, pp. 547-550, Padova, Italy, July 1998.

[4] G. Weiss and R. Rebarber, "Optimizability and estimatability for infinite-dimensional linear systems," SIAM Journal on Control and Optimization, vol. 39, no. 4, pp. 1204-1232, 2000.

[5] K. M. Mikkola, "Coprime factorization and dynamic stabilization of transfer functions," SIAM Journal on Control and Optimization, vol. 45, no. 6, pp. 1988-2010, 2007.

[6] R. F. Curtain, G. Weiss, and M. Weiss, "Stabilization of irrational transfer functions by controllers with internal loop, Systems, approximation, singular integral operators, and related topics," in Proceedings of the International Workshop on Operator Theory and Applications (IWOTA), A. A. Borichev and N. K. Nikolski, Eds., vol. 129 of Advances in Operator Theory and Applications, pp. 179-207, Birkhäauser, Basel, 2000.

[7] R. F. Curtain, "Robustly stabilizing controllers with internal loop," in Control of uncertain systems: modelling, approximation, and design, vol. 329 of Lecture Notes in Control and Inform. Sci., pp. 79-98, Springer, Berlin, Germany, 2006.

[8] A. Feintuch, Robust Control Theory in Hilbert Space, vol. 130, Springer, New York, NY, USA, 1998.

[9] L. Ma, Z. Wang, Y. Bo, and Z. Guo, "A game theory approach to mixed $\mathscr{L}_{2} / \mathscr{L}_{\infty}$ control for a class of stochastic time-varying systems with randomly occurring nonlinearities," Systems and Control Letters, vol. 60, no. 12, pp. 1009-1015, 2011.

[10] J. Hu, Z. Wang, H. Gao, and L. K. Stergioulas, "Probability-guaranteed $H_{\infty}$ finite-horizon filtering for a class of nonlinear time-varying systems with sensor saturations," Systems and Control Letters, vol. 61, no. 4, pp. 477-484, 2012.

[11] G. Wei, Z. Wang, and B. Shen, "Error-constrained finite-horizon tracking control with incomplete measurements and bounded noises," International Journal of Robust and Nonlinear Control, vol. 22, no. 2, pp. 223-238, 2012.

[12] B. Shen, Z. Wang, H. Shu, and G. Wei, " $H_{\infty}$ filtering for uncertain time-varying systems with multiple randomly occurred nonlinearities and successive packet dropouts," International Journal of Robust and Nonlinear Control, vol. 21, no. 14, pp. 1693-1709, 2011.

[13] B. Shen, Z. Wang, and X. Liu, "Bounded $H_{\infty}$ synchronization and state estimation for discrete timevarying stochastic complex networks over a finite horizon," IEEE Transactions on Neural Networks, vol. 22, no. 1, Article ID 5640676, pp. 145-157, 2011.

[14] G. Wei, Z. Wang, and B. Shen, "Error-constrained filtering for a class of nonlinear time-varying delay systems with non-Gaussian noises," IEEE Transactions on Automatic Control, vol. 55, no. 12, pp. 28762882, 2010. 
[15] Z. Wang, D. W. C. Ho, H. Dong, and H. Gao, "Robust $\mathscr{\ell}_{\infty}$ finite-horizon control for a class of stochastic nonlinear time-varying systems subject to sensor and actuator saturations," IEEE Transactions on Automatic Control, vol. 55, no. 7, pp. 1716-1722, 2010.

[16] H. Dong, Z. Wang, D. W. C. Ho, and H. Gao, "Variance-constrained $\mathscr{H}_{\infty}$ filtering for a class of nonlinear time-varying systems with multiple missing measurements: the finite-horizon case," IEEE Transactions on Signal Processing, vol. 58, no. 5, pp. 2534-2543, 2010.

[17] A. Feintuch, "On strong stabilization for linear time-varying systems," Systems $\mathcal{E}$ Control Letters, vol. 54, no. 11, pp. 1091-1095, 2005. 


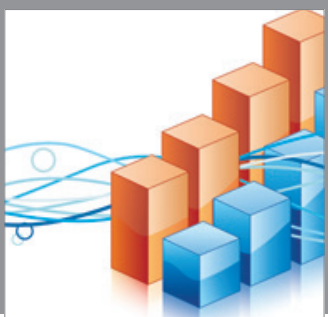

Advances in

Operations Research

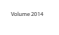

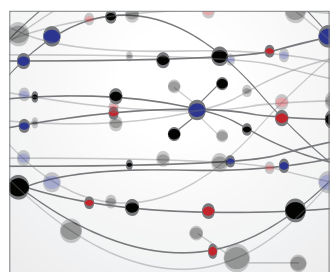

\section{The Scientific} World Journal
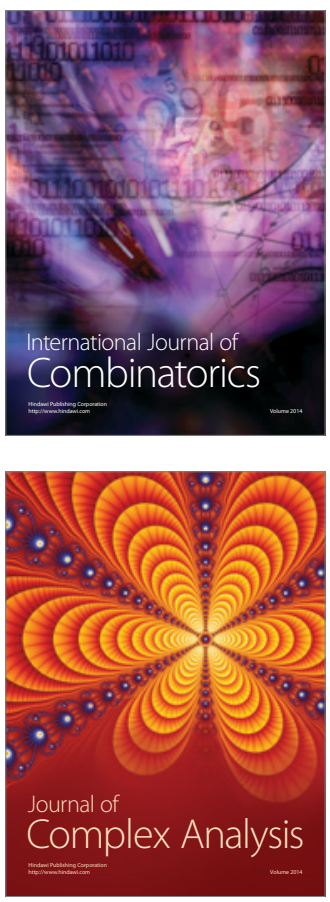

International Journal of

Mathematics and

Mathematical

Sciences
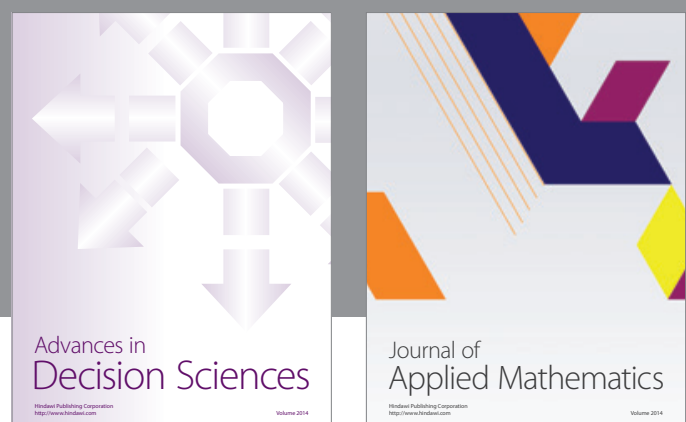

Journal of

Applied Mathematics
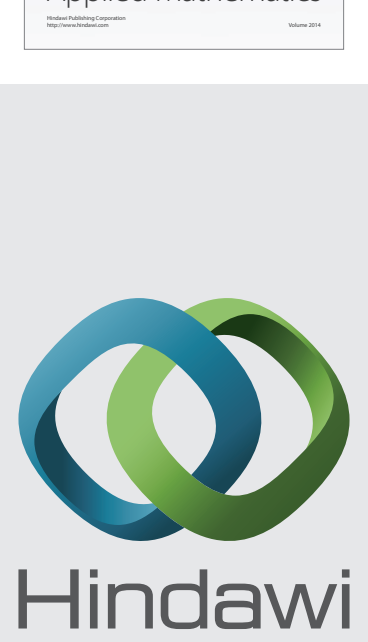

Submit your manuscripts at http://www.hindawi.com
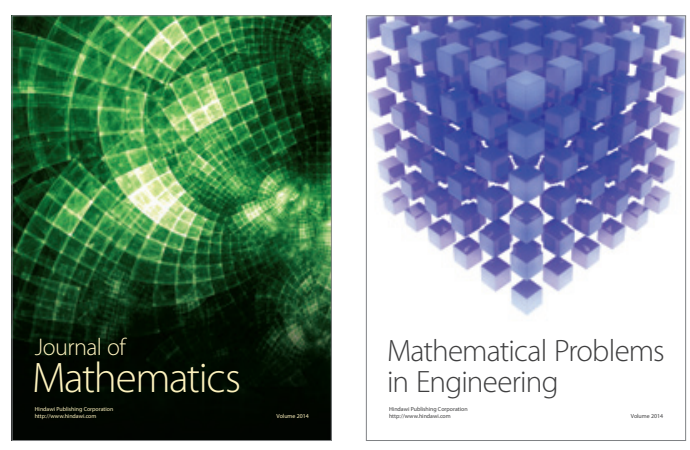

Mathematical Problems in Engineering
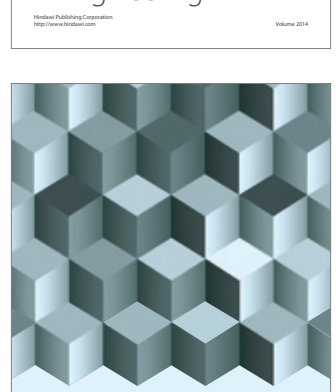

Journal of

Function Spaces
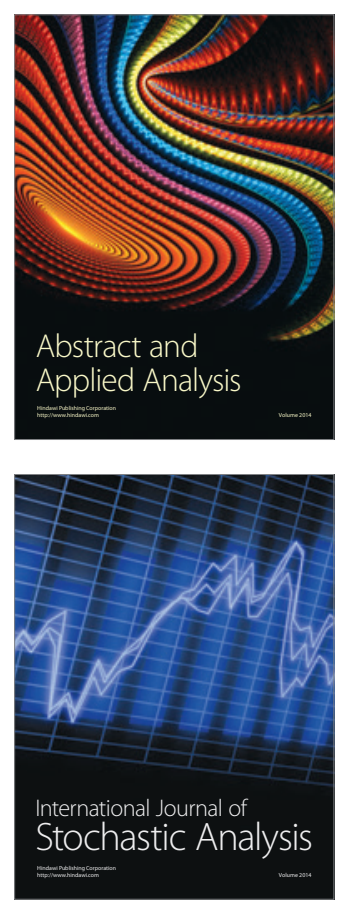

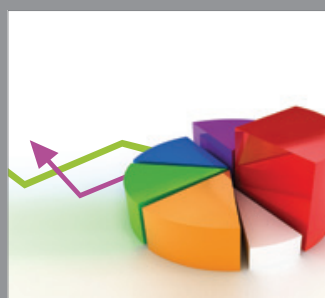

ournal of

Probability and Statistics

Promensencen
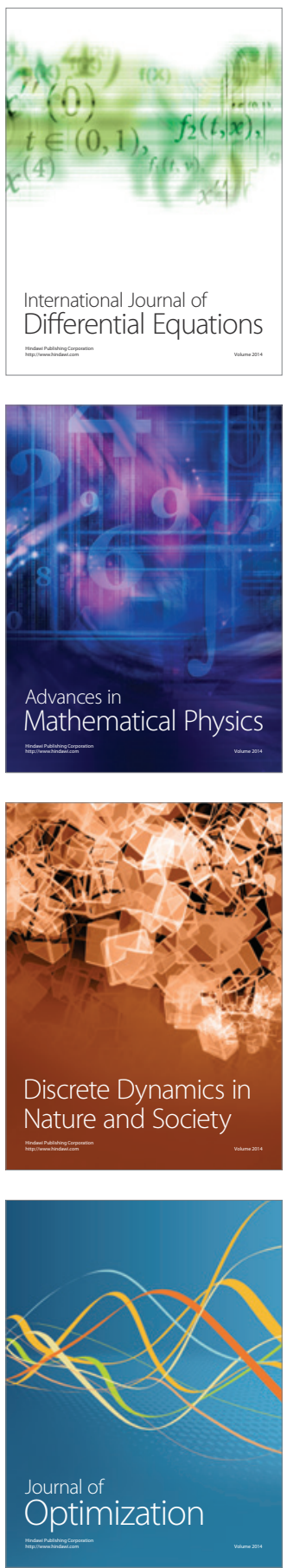